\title{
Pulmonary and pleural metastasis of intracranial anaplastic meningioma in a 3-year-old boy: A case report
}

\author{
YUKO HONDA, RIE SHIRAYAMA, HIROMI MORITA and KOICHI KUSUHARA \\ Department of Pediatrics, University of Occupational and Environmental Health, Kitakyushu, Fukuoka 807-8555, Japan
}

Received March 16, 2017; Accepted August 7, 2017

DOI: $10.3892 / \mathrm{mco} .2017 .1375$

\begin{abstract}
In adults, meningiomas occasionally display aggressive behavior and may occasionally metastasize. By contrast, pediatric meningiomas are rare, and there is limited information regarding their clinical characteristics, treatment and prognosis. We herein report the case of a 3-year-old boy with anaplastic meningioma with a history of local recurrence and late pulmonary metastasis. At diagnosis, a 70-mm mass lesion in was identified in the right frontal lobe, with intratumoral hemorrhage. The tumor was attached to the falx cerebri and was completely resected. The histological diagnosis was anaplastic meningioma, World Health Organization grade III. Two months after the surgery, the meningioma recurred at the same site. Although the patient received radiotherapy after a second operation, the tumor metastasized to the lung and pleura 8 months after the initial operation. The metastasis was resistant to treatment, even after gross total resection, and the effectiveness of further radiotherapy was limited. The patient succumbed to the disease 1 year and 4 months after the initial diagnosis. The findings of the present case and a review of the relevant literature suggest that recurrence and metastasis of meningiomas are difficult to predict. Therefore, such patients should be carefully monitored throughout the follow-up period.
\end{abstract}

\section{Introduction}

Intracranial meningiomas, the most common central nervous system (CNS) tumors, account for $\sim 30 \%$ of all CNS tumors in adults, whereas pediatric intracranial meningiomas are rare, comprising only $0.4-4.6 \%$ of all pediatric primary intracranial tumors $(1,2)$. As pediatric intracranial meningiomas are rare, there is little available information on their epidemiology, treatment and prognosis.

Correspondence to: Dr Yuko Honda, Department of Pediatrics, University of Occupational and Environmental Health, 1-1 Iseigaoka, Yahatanishi-Ku, Kitakyushu, Fukuoka 807-8555, Japan

E-mail: h-yukoc@med.uoeh-u.ac.jp

Key words: anaplastic meningioma, lung metastasis, malignant meningioma, childhood, oncology
Pediatric intracranial meningiomas are characterized by the presence of uncommon lesions within the intraventricular or intraorbital locations. They are slightly more predominant in males and appear to be more aggressive compared with adult cases of meningioma. In the present case report, we present the clinical course of a 3 -year-old boy with anaplastic meningioma who developed extracranial metastases.

\section{Case report}

A 3-year-old boy was admitted to the Department of Pediatrics (University of Occupational and Environmental Health, Kitakyushu, Japan) in April 2011, due to vomiting, fever and left-side weakness that had lasted for 10 days. A brain computed tomography (CT) scan revealed an isodense mass lesion in the right frontal lobe with severe peritumoral brain edema. Magnetic resonance imaging (MRI) revealed a 70-mm mass lesion in the right frontal lobe with heterogeneous intensity associated with intratumoral hemorrhage (Fig. 1). On the day after his admission, the patient's consciousness progressively deteriorated. Emergency surgery was thus performed and the tumor was removed in its entirety. The histological diagnosis was anaplastic meningioma, World Health Organization (WHO) grade III, and the MIB-1 labeling index was $25.4 \%$.

Two months after the first operation, an MRI of the head revealed a local recurrence at the same site as the initial tumor. Thus, a second operation was performed and the tumor was completely removed. However, 10 days later, multiple tumors were detected in other areas of the brain. Radiotherapy (6,120 cGy, frontal region irradiation) was then administered, resulting in disappearance of the recurrent tumors. The patient had no neurological defects, and no further tumors were detected over the subsequent 4 months, throughout which regular MRI examinations were conducted.

At 8 months after the initial operation, the patient developed cough, fever, chest pain, and had difficulty breathing. A CT scan of the thorax revealed a large mass $(\sim 85 \mathrm{~mm})$ with pleural effusion in the right lung (Fig. 2A). As there was no evidence of other local recurrence or malignant lesions in any other organs, a right middle and lower lobectomy was performed. Tissue specimens obtained from the lung revealed that the tumor was well-demarcated from the adjacent lung parenchyma and exhibited focal necrotic and hemorrhagic changes. The tumor cells had oval nuclei with a high nuclear-cytoplasmic ratio and large, prominent nucleoli (Fig. 3). Immunohistochemical 
examination revealed strong expression of anti-cytokeratin (CAM5.2) and CD99; furthermore, the MIB-1 (Ki-67) proliferation index was $80 \%$. Based on these findings, the diagnosis was lung metastasis of anaplastic meningioma, in agreement with the pathological characteristics of the primary intracranial anaplastic meningioma. The tumor was then completely surgically removed; however, multiple pleural-based tumors were detected during a follow-up MRI 1 month after the lobectomy (Fig. 2B) and local radiotherapy was administered. Although the pleural-based tumors disappeared following administration of radiotherapy (Fig. 2C), they reappeared shortly after the completion of radiation therapy in the right lung and right frontal lobe. In response to this finding, 4 additional courses of radiotherapy were administered for these lesions. Unfortunately, the radiotherapy was ultimately unsuccessful in the treatment of these metastases and the patient succumbed to the disease $\sim 1$ year and 4 months after the initial diagnosis. The patient's family has consented to the publication of the case details and associated images.

\section{Discussion}

As cases of pediatric intracranial meningioma are rare, only small single-center and retrospective studies of these neoplasms have been published to date (1-3). Furthermore, all these studies have consistently found that the clinical and biological aspects of pediatric intracranial meningioma cases differ from those of adult cases.

According to the WHO classification system, there are 15 subtypes of meningioma (4). In adults, $290 \%$ of meningiomas are benign (grade I), with $5-7 \%$ classified as aggressive (grade II) and the remaining 1-3\% graded as malignant (grade III) (5). By contrast, higher grades are more common in pediatric meningiomas. A meta-analysis determined the incidence of WHO grade II and III meningiomas to be 9.9 and $8.9 \%$, respectively. Furthermore, it has been reported that WHO grade III pediatric meningioma cases, had a significantly worse relapse-free survival (RFS) rate compared with WHO grade I tumors (5-year RFS rates: 40.7 vs. 81.2\%, respectively). However, the WHO grading system does not account for the overall survival rate for pediatric meningiomas (6). Thus, the biological behavior of pediatric meningiomas is notoriously difficult to predict on the basis of the WHO grade alone.

Kotecha et al reported that the extent of the initial surgical resection was the strongest independent prognostic factor (6). However, some reports indicated that recurrence was very common for high-grade lesions, even following complete resection $(1,7,8)$. Our case is consistent with these latter reports, as, although gross total excision of the tumor was possible, the tumor reappeared in the same location 2 months later.

The MIB-1 index is an important prognostic factor for meningiomas. In adults, the MIB-index is well-correlated with the histological grade and the rate of tumor recurrence. However, conversely, there is a lack of corresponding data on pediatric meningiomas due to the limited number of cases. Wang et al reported that the association between the MIB-1 index and the pathological grade was generally weak for intracranial pediatric meningiomas. Moreover, pediatric meningiomas with an MIB-1 index $>3 \%$ appeared to have a worse prognosis (9). In the present case, the patient's MIB-1
$\mathbf{A}$ B

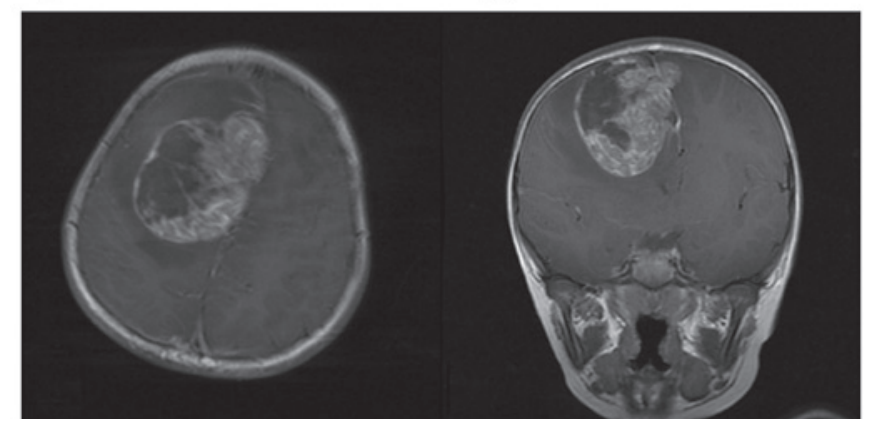

Figure 1. Brain magnetic resonance imaging at diagnosis (A) Axial contrast-enhanced T1-weighted images. (B) Coronal view. The tumor was enhanced heterogeneously following gadolinium administration.

index was relatively high at diagnosis and increased progressively, reaching $80 \%$ at the lung metastasis stage. This progression of the MIB-1 index was associated with the proliferative potential of the tumor and the time interval to the next recurrence or lung metastasis. For malignant meningiomas, it is difficult to make a reliable prognosis on the basis of the MIB-1 index at the time of diagnosis, or based on the results of surgical resection alone. Therefore, pediatric patients with malignant meningioma or a high MIB-1 index must be carefully monitored, as physicians must be aware of the possibility of an extracranial metastasis in such cases.

Although metastasis of meningioma is a rare phenomenon in adult patients, it has been well-documented and its incidence has been estimated to be $0.1 \%(10,11)$. According to the results of the systematic review by Surov et al (12), metastatic lesions were localized most frequently in the lungs (37.2\%), bones $(16.5 \%)$, intraspinally $(15.2 \%)$ and in the liver $(9.2 \%)$. Unlike adult cases, metastatic meningioma is extremely rare in pediatric cases; thus, there are only few reports on extracranial metastases $(6,12)$ and the corresponding rate has not yet been determined. To the best of our knowledge, prior to our report, a total of 4 cases of pediatric meningioma with lung metastases have been published (Table I) and our report presents the youngest such patient. Moreover, all 5 cases, including ours, were classified as WHO grade III, and the mean patient age was 9 years (range, 3-14 years). Although the intracranial tumors were completely resected in 3 of the 5 cases $(60 \%)$, they all recurred. The time interval between the initial diagnosis and the occurrence of lung metastases ranged from 8 to 18 months. All the patients succumbed to lung metastasis, and their survival times ranged from 8 to 30 months. Therefore, based on the present case and the few preceding reported cases, it is our conclusion that meningioma cases that progress to metastasis are very resistant to treatment, even following gross total resection.

There is currently no standard treatment for metastatic meningioma, and there are no particularly effective medications for this disease. Although surgical excision is the treatment of first choice for pediatric and adult meningiomas, complete tumor resection cannot prevent recurrence or metastasis. Furthermore, radiotherapy is currently considered by the National Institute for Health and Clinical Excellence to be a plausible form of treatment for adults with WHO 


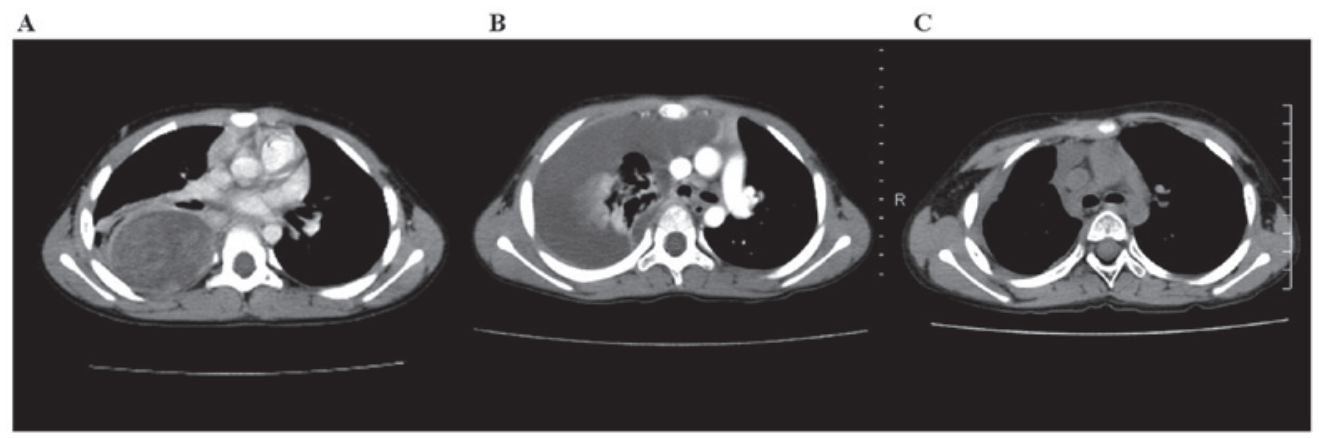

Figure 2. An enhanced computed tomography scan of the chest revealed a pleural-based mass in the right lung. (A) Image obtained 8 months after the initial intracranial operation. (B) Image obtained 1 month after right lung lobectomy. (C) Image obtained immediately following radiotherapy.
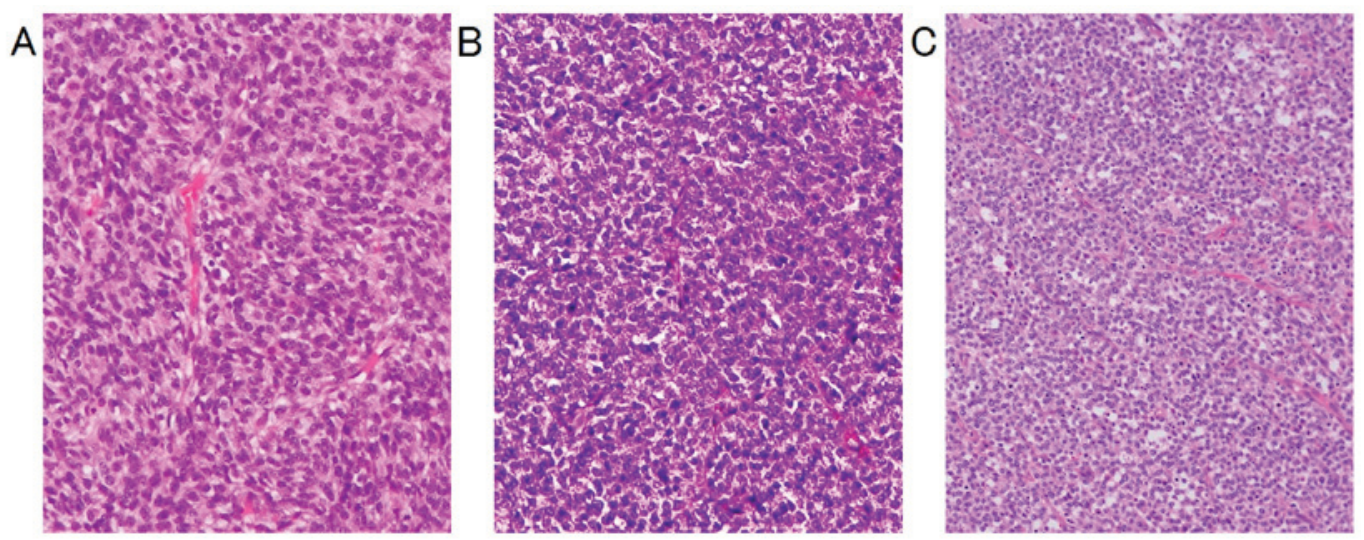

Figure 3. On histological examination, the tumor exhibited increased cellularity, small cells with a high nucleus/cytoplasm ratio and prominent nucleoli, arranged in a sheet-like pattern. (A) Primary meningioma, (B) recurrent meningioma, (C) pulmonary metastasis. Hematoxylin and eosin staining; magnification, x10.

Table I. Characteristics of pediatric meningiomas that developed lung metastases.

\begin{tabular}{clllcccc}
\hline Age, sex & \multicolumn{1}{c}{ Location at Dx } & Pathology & GTR & Recurrence & $\begin{array}{c}\text { Time interval between } \\
\text { Dx and lung metastasis }\end{array}$ & $\begin{array}{c}\text { Outcome } \\
\text { (months) }\end{array}$ & (Refs.) \\
\hline 5,f & Right occipital & Papillary & Yes & Yes & 18 months & Deceased (23) & $(9)$ \\
9, m & Right parietal & Anaplastic & No & Yes & 8 months & Deceased (8) & $(9)$ \\
12, f & Parasagittal & Malignant & ND & Yes & ND & Deceased (14) & $(2)$ \\
14, m & Parietal scalp and skull & Malignant & Yes & Yes & ND & Deceased (30) & $(13)$ \\
3, m & Right frontal & Anaplastic & Yes & Yes & 9 months & Deceased (15) & Present case \\
\hline
\end{tabular}

f, female; m, male; Dx, diagnosis; GTR, gross total resection; metastasis; ND, no data.

grade II or III tumors, multiple relapses, contraindication to surgery, invasion of adjacent brain, or extensive invasion of other tissues (14). Conversely, according to the Children's Cancer and Leukemia Group (CCLG) guidelines, the data supporting the use of adjuvant radiotherapy for pediatric high-risk cases are scant; however, radiotherapy should be considered at the time of the primary diagnosis, regardless of the surgical outcome, particularly in cases of WHO grade III anaplastic meningioma (15).

In the present case, the patient ultimately succumbed to disease progression, despite radiotherapy treatment. However, it should be noted that radiotherapy was somewhat effective initially in preventing local recurrence and it also prolonged the patient's survival time. Furthermore, no further intracranial tumors were detected even 2 months after the occurrence of metastases to the lungs, and the patient survived for a further 7 months after the metastases. Therefore, although there is no conclusive data regarding the benefit of adjuvant radiotherapy in pediatric meningioma cases, it may be associated with some benefits in malignant cases of pediatric meningioma.

Furthermore, although the mechanism of pulmonary metastases from intracranial meningiomas is unclear, it is thought that the main dissemination pathway is through the CSF and venous sinus invasion $(12,16)$. If surgical resection of a tumor has the potential to damage the blood brain barrier and, thus, instigate distant metastasis, radiotherapy may be 
useful at the time of primary diagnosis in WHO grade III pediatric anaplastic meningioma cases.

To reiterate, the number of cases of pediatric meningioma are extremely rare and only small single-center and retrospective studies are currently available. Thus, in order to assess the positive effects of radiotherapy, prospective clinical trials are required.

In conclusion, the occurrence of metastatic meningiomas is possible, although rare, in young children. Furthermore, high-grade pediatric meningiomas are associated with high recurrence and mortality rates, and there are currently no definitive criteria for estimating the probability of recurrence or metastasis. Therefore, such patients should be closely monitored throughout the follow-up period. Moreover, due to the rarity of pediatric meningioma, there is currently no standard treatment for metastatic meningioma. Thus, the true incidence and impact of distant metastases in this subset of patients should be corroborated through prospective clinical trials.

\section{References}

1. Ravindranath K, Vasudevan MC, Pande A and Symss N: Management of pediatric intracranial meningiomas: An analysis of 31 cases and review of literature. Childs Nerv Syst 29: 573-582, 2013

2. Rushing EJ, Olsen C, Mena H, Rueda ME, Lee YS, Keating RF, Packer RJ and Santi M: Central nervous system meningiomas in the first two decades of life: A clinicopathological analysis of 87 patients. J Neurosurg 103 (6 Suppl): S489-S495, 2005.

3. Lakhdar F, Arkha Y, EI Ouahabi A, Melhaoui A, Rifi L, Derraz $\mathrm{S}$ and El Khamlichi A: Intracranial meningioma in children: Different from adult forms? A series of 21 cases. Neurochirurgie 56: 309-314, 2010.

4. Louis DN, Ohgaki H, Wiestler OD, Cavenee WK, Burger PC, Jouvet A, Scheithauer BW and Kleihues P: The 2007 WHO classification of tumours of the central nervous system. Acta Neuropathol 114: 97-109, 2007.

5. Commins DL, Atkinson RD and Burnett ME: Review of meningioma histopathology. Neurosurg Focus 23: E3, 2007.
6. Kotecha RS, Pascoe EM, Rushing EJ, Rorke-Adams LB, Zwerdling T, Gao X, Li X, Greene S, Amirjamshidi A, Kim SK, et al: Meningiomas in children and adolescents: A meta-analysis of individual patient data. Lancet Oncol 12: 1229-1239, 2011

7. Arivazhagan A, Devi BI, Kolluri SV, Abraham RG, Sampath S and Chandramouli BA: Pediatric intracranial meningiomas-do they differ from their counterparts in adults? Pediatr Neurosurg 44: 43-48, 2008.

8. Greene S, Nair N, Ojemann JG, Ellenbogen RG and Avellino AM: Meningiomas in children. Pediatr Neurosurg 44: 9-13, 2008.

9. Wang XQ, Jiang CC, Zhao L, Gong Y, Hu J and Chen H: Clinical features and treatment of World Health Organization grade II and III meningiomas in childhood: Report of 23 cases. J Neurosurg Pediatr 10: 423-433, 2012.

10. Alexandru D, Glantz MJ, Kim L, Chamberlain MC and Bota DA: Pulmonary metastases in patients with recurrent, treatment-resistant meningioma: Prognosis and identification by ${ }^{111}$ Indium-octreotide imaging. Cancer 117: 4506-4511, 2011.

11. Rhim JK, Sheen SH, Noh JS and Chung SB: Pulmonary metastasis of malignant meningioma. J Korean Neurosurg Soc 35: 533-535, 2004

12. Surov A, Gottschling S, Bolz J, Kornhuber M, Alfieri A, Holzhausen HJ, Abbas J and Kösling S: Distant metastases in meningioma: An underestimated problem. J Neurooncol 112: 323-327, 2013.

13. Baumgartner JE and Sorenson JM: Meningioma in the pediatric population. J Neurooncol 29: 223-228, 1996.

14. National Institute for Health and Clinical Excellence: Improving Outcomes for People with Brain and Other CNS Tumours: Guidance on Cancer Services. NICE http://www.nice.org. uk/guidance/csgbraincns/evidence/improving-outcomes-for-pe ople-with-brain-and-other-cns-tumours-the-manual2. Accessed March 20,2015.

15. Traunecker H, Mallucci C, Grundy R, Pizer B and Saran F; Children's Cancer and Leukaemia Group: Children's Cancer and Leukaemia Group (CCLG): Guidelines for the management of intracranial meningioma in children and young people. Br J Neurosurg 22: 13-25, 2008.

16. Forest F, Berremila SA, Gyenes C, Ginguéné C, Kassir R, Sulaiman A, Pasquier B, Porcheron J and Péoc'h M: Metastatic meningiomas: An unusual clinical and pathological diagnosis with highly variable outcome. J Neurooncol 120: 411-421, 2014. 